\title{
Challenges in Referral Communication Between VHA Primary Care and Specialty Care
}

\author{
Jessica L. Zuchowski, PhD, MPH' , Danielle E. Rose, $P h D, M P H^{7}$, Alison B. Hamilton, $P h D, M P H^{1,2}$, \\ Susan E. Stockdale, $P h D^{1,2}$, Lisa S. Meredith, $P h D^{7,3}$, Elizabeth M. Yano, PhD, MSPH, \\ Lisa V. Rubenstein, MD, MSPH, FACP ${ }^{1,3,5}$, and Kristina M. Cordasco, MD, MPH, MSHS ${ }^{1,5}$
}

\begin{abstract}
${ }^{1}$ Center for the Study of Healthcare Innovation, Implementation and Policy, VA Greater Los Angeles Healthcare System, Sepulveda, CA, USA; ${ }^{2}$ Department of Psychiatry and Biobehavioral Sciences, David Geffen School of Medicine, UCLA, Los Angeles, CA, USA; ${ }^{3}$ RAND Corporation, Santa

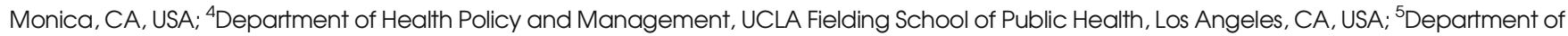
Medicine, David Geffen School of Medicine, UCLA, Los Angeles, CA, USA.
\end{abstract}

BACKGROUND: Poor communication between primary care providers (PCPs) and specialists is a significant problem and a detriment to effective care coordination. Inconsistency in the quality of primary-specialty communication persists even in environments with integrated delivery systems and electronic medical records (EMRs), such as the Veterans Health Administration (VHA).

OBJECTIVE: The purpose of this study was to measure ease of communication and to characterize communication challenges perceived by PCPs and primary care personnel in the VHA, with a particular focus on challenges associated with referral communication.

DESIGN: The study utilized a convergent mixed-methods design: online cross-sectional survey measuring PCPreported ease of communication with specialists, and semi-structured interviews characterizing primary-specialty communication challenges.

PARTICIPANTS: 191 VHA PCPs from one regional network were surveyed (54\% response rate), and 41 VHA PCPs and primary care staff were interviewed.

MAIN MEASURES/APPROACH: PCP-reported ease of communication mean score (survey) and recurring themes in participant descriptions of primary-specialty referral communication (interviews) were analyzed.

KEY RESULTS: Among PCPs, ease-of-communication ratings were highest for women's health and mental health (mean score of 2.3 on a scale of 1-3 in both), and lowest for cardiothoracic surgery and neurology (mean scores of 1.3 and 1.6, respectively). Primary care personnel experienced challenges communicating with specialists via the EMR system, including difficulty in communicating special requests for appointments within a certain time frame and frequent rejection of referral requests due to rigid informational requirements. When faced with these challenges, PCPs reported using strategies such as telephone and e-mail contact with specialists with whom they had established relationships, as well as the use of an EMR-based referral innovation called "eConsults" as an alternative to a traditional referral.

Received April 8, 2014

Revised October 3, 2014

Accepted October 29, 2014

Published online November 20, 2014
CONCLUSIONS: Primary-specialty communication is a continuing challenge that varies by specialty and may be associated with the likelihood of an established connection already in place between specialty and primary care. Improvement in EMR systems is needed, with more flexibility for the communication of special requests. Building relationships between PCPs and specialists may also facilitate referral communication.

KEY WORDS: primary care; specialty care; communication; electronic health records; veterans.

J Gen Intern Med 30(3):305-11

DOI: $10.1007 / \mathrm{s} 11606-014-3100-\mathrm{x}$

(C) Society of General Internal Medicine 2014

\section{BACKGROUND}

A growing body of literature suggests that poor primary-specialty communication is a significant problem within the U.S. healthcare system and a detriment to effective care coordination. ${ }^{1-10}$ Studies have found high rates of dissatisfaction with different aspects of primary-specialty communication-and specifically referral communication. ${ }^{4,5}$ This problem is of increasing concern, as there have been recent national trends towards proportionally more primary care visits resulting in referrals to specialty care. ${ }^{11}$ Optimal communication between primary and specialty providers is essential for realizing the goals of the patient-centered medical home (PCMH) care mod$\mathrm{el}$, an increasingly dominant paradigm in primary care throughout the country. ${ }^{12}$

Integrated delivery systems and shared electronic medical records (EMRs) can facilitate communication between primary and specialty care providers. ${ }^{8}$ An integrated delivery system is more likely to have an infrastructure of operational agreements already in place between primary and specialty services, an incentive structure that supports communication outside the office visit setting, and a sense of shared mission among providers. ${ }^{13-16}$ The use of a single, shared EMR is cited in a number of studies as an effective means of conveying patients' complete medical histories from primary to specialty care, as well as communicating specialists' assessments back to primary care. ${ }^{8,15,17-20}$ 
EMRs can also be applied specifically to facilitate the referral process, one of the most common and important types of primary-specialty care communication. ${ }^{21,22}$

The Veterans Health Administration (VHA) is a fully integrated healthcare system with a well-developed, comprehensive EMR system. The VHA's EMR is the dominant method of connecting primary and specialty care at the VHA, serving as the vehicle for conveying requests from primary care for specialty consults as well as specialist recommendations back to primary care. One study conducted within the VHA, however, identified a subset of cases evidencing unexplained breakdowns in primary-specialty referral communication. ${ }^{21}$ The EMR alone, therefore, may not be "enough" to solve the problems in primary-specialty communication. ${ }^{4,9,13,23}$

Little is known about referral communication between primary and specialty care in the VHA and similar healthcare systems that already benefit from an integrated delivery system and shared EMRs. More specifically, there is a lack of information about the nature of primary-specialty communication challenges, including potential specialty-dependent variability, as well as primary care provider (PCP) responses to these challenges. We used survey and interview data from VHA clinicians and leadership to explore these issues.

\section{METHODS}

\section{Setting}

This study was conducted within three southern California VHA healthcare systems. Data were collected through the Veterans Assessment and Improvement Laboratory for Patient-Centered Care (VAIL), one of five demonstration labs funded by the VHA's Office of Patient Care Services to evaluate the VHA's approach to PCMH primary care transformation, the Patient Aligned Care Team (PACT) model. ${ }^{24}$ The purpose of VAIL was to test evidence-based quality improvement as a method for developing and promoting a culture of quality improvement in primary care settings. ${ }^{25}$ All procedures were approved by the Greater Los Angeles VHA Institutional Review Board (IRB), and the survey was also approved by the RAND IRB.

\section{Quantitative Procedures}

To measure PCP-reported ease of communication with specialists, we analyzed data from a cross-sectional Internetbased survey of all PCPs in one regional network, which was administered by the RAND Corporation and fielded from November 29, 2011, through March 9, 2012. Items for the present analysis were drawn from a baseline survey assessing the VHA's PCMH implementation. Items were developed by a multidisciplinary team comprising content experts in clinical medicine, psychology, implementation science, and organizational behavior. Many of the item sets were from published scales that had been previously validated. Items that were developed specifically for this study were pretested and validated on a small sample of PCPs and other primary care staff.

Response options to the measure were two-pronged. First, we asked whether PCPs communicated with the various specialty providers; if the answer was yes, we asked them to rate the communication. PCPs were asked, "How easy is it for you to communicate [in person, by phone, or electronically] with the following types of health services providers?" The response list included medical subspecialists (e.g., cardiologists, nephrologists), surgical specialists (e.g., general surgery, cardiothoracic surgery), population specialists (e.g., geriatrics, women's health), and other specialists (e.g., neurology, rehabilitation, mental/behavioral health). Response options, on a three-point Likert scale, ranged from "very easy" to "somewhat easy" to "not at all easy."

\section{Qualitative Procedures}

To investigate primary-specialty communication challenges, we interviewed PCPs and primary care staff at three sites within the same regional network as that in the survey. Interviewees, identified by job title and role in PACT implementation, were recruited via e-mail, and included primary care team members (PCPs, registered nurses [RNs], licensed vocational nurses [LVNs], and clerks). Our interdisciplinary research team developed the interview guide in accordance with the project's goals and conceptual model. ${ }^{25}$ The guide was designed to elucidate ground-level processes around various aspects of PACT implementation, including connections with specialty care, from the perspective of all four primary care roles. We asked primary care team members: "Are you involved in coordinating care between your team and specialist physicians? Who on your team is involved in coordinating with specialists? Can you tell me about the most significant specialist communication or coordination change or improvement? To your knowledge, does your team face challenges communicating or coordinating with any specialist physicians or services? Can you tell me about this?" Interviews were audio-recorded, professionally transcribed, and spot-checked for accuracy.

\section{Quantitative Analysis}

Based on primary care providers' ratings of ease of communication, we calculated a mean score (very easy $=3$, somewhat easy $=2$, or not at all easy $=1$ ) for each type of specialist and specialist group. We tested for differences among specialist group scores in two ways. First, we performed an F-test to determine whether there were any statistically significant differences among the four groups. After rejecting the null hypothesis that they were all equal, we conducted $t$ tests for each pair of specialist groups. To account for survey non-response, we calculated and applied non-responder weights based on job title and site. 


\section{Qualitative Analysis}

To identify transcripts that contained data pertinent to primary-specialty care communication, we manually reviewed all transcripts for four separate truncated keywords: "special" (which captured references to specialists and specialty care), "refer" (which captured references to referrals), "consult" (the short term used to describe a specialty consultation request placed in the VHA's EMR), and "mental" (which captured references to the integration of mental health services into primary care). Each instance of one or more of these keywords was individually evaluated for relevance and depth of content. Interviews with relevant content were then reviewed in their entirety and relevant sections coded for emergent themes that appeared repeatedly in the data, such as appointment scheduling, denied consults, and EMR. To confirm manual transcript review, we used ATLAS.ti version 6 software (Scientific Software Development $\mathrm{GmbH}$, Berlin, Germany) to identify the same four keywords in all transcripts. This output was reviewed and cross-checked by a second coder. In addition, all interviews were coded for content related to primaryspecialty communication and checked by a second coder. We synthesized results using clustering of coded segments by emergent themes around primary-specialty communication and by respondent role in order to examine whether there was variability in perceptions of communication by type of respondent.

\section{RESULTS}

\section{Survey}

Of the 356 people invited, 191 (54\%) PCPs participated in the survey and responded to our questions of interest. The means and standard deviations for each type of specialist and specialist group (medical subspecialists, surgical specialists, population specialists, and other specialists) are presented in Table 1. PCPs reported the highest ease-of-communication scores with mental health and women's health (mean score $2.3 \pm 0.7$ for both types of specialists), and the lowest scores with cardiothoracic surgery and neurology (mean scores of 1.3 \pm 0.5 and $1.6 \pm 0.7$, respectively). When results were averaged by specialty group, PCPs reported the highest ease of communication with population specialists (mean score $2.1 \pm 0.6$ ), and lowest scores with surgical specialists (mean score $1.7 \pm$ 0.5 ). We rejected the null hypothesis that the groups of specialists were equal (F-test $<.0000)$. When we tested groups' scores for statistically significant differences, adjusting for multiple comparisons, we found statistically significant differences in communication scores for all pairs of specialist groups but one: medical subspecialists and other specialists (Table 2). Of the 191 participants, 2-4\% did not answer an item in the measure; the percentage of participants reporting no communication with a specialty ranged from $2 \%$ to $29 \%$, based on the specialty. When we dropped from the analysis the
Table 1 VHA Primary Care Provider (PCP) Ratings of Ease of Communication with Specialists by Specialist Type $(n=191)$

\begin{tabular}{|c|c|c|}
\hline & \multicolumn{2}{|c|}{$\begin{array}{l}\text { PCP rating of ease of } \\
\text { communication with specialists* }\end{array}$} \\
\hline & Mean & Standard Deviation \\
\hline \multicolumn{3}{|l|}{ Medical subspecialists } \\
\hline Cardiology & 1.9 & 0.7 \\
\hline Nephrology & 1.8 & 0.7 \\
\hline Oncology & 1.7 & 0.7 \\
\hline Endocrinology & 1.9 & 0.7 \\
\hline Gastroenterology & 1.9 & 0.7 \\
\hline Pulmonary & 1.8 & 0.7 \\
\hline Infectious diseases & 2.1 & 0.7 \\
\hline Average & 1.9 & 0.6 \\
\hline \multicolumn{3}{|l|}{ Surgical specialists } \\
\hline General surgery & 1.7 & 0.7 \\
\hline Cardiothoracic surgery & 1.3 & 0.5 \\
\hline Orthopedics & 1.7 & 0.7 \\
\hline Ophthalmology & 1.8 & 0.7 \\
\hline Urology & 1.9 & 0.7 \\
\hline Average & 1.7 & 0.5 \\
\hline \multicolumn{3}{|l|}{ Population specialists } \\
\hline Geriatrics & 1.9 & 0.8 \\
\hline Women's health & 2.3 & 0.7 \\
\hline HIV/AIDS & 2.1 & 0.7 \\
\hline Average & 2.1 & 0.6 \\
\hline \multicolumn{3}{|l|}{ Other specialists } \\
\hline Neurology & 1.6 & 0.7 \\
\hline Rehabilitation & 2.0 & 0.7 \\
\hline Mental/Behavioral health & 2.3 & 0.7 \\
\hline Average & 2.0 & 0.5 \\
\hline
\end{tabular}

"Primary care providers who reported communicating with specialty providers were asked, "How easy it is for you to communicate [in person, by phone, or electronically] with the following types of health services providers?" Response options ranged from very easy (3) to somewhat easy (2) to not at all easy (1). We calculated the mean score for each type of specialist, and then calculated means for each specialist group. The means and standard deviations for each specialist type and specialist group are presented here.

two specialists for which the highest percentage of participants reported no communication, it had no impact on the outcomes of any of the tests.

\section{Interviews}

Of 72 primary care team members who were invited to be interviewed, semi-structured interviews were conducted with 39 (54\%) team members between February 2012 and June 2012; these included 14 PCPs, 9 RNs, 9 LVNs, and 7 clerks. Our initial analysis revealed that $32(82 \%)$ of the interviews contained content relevant to primary-specialty communication,

Table 2 Comparison of Primary Care Providers' Ratings of Ease of Communication with Specialists by Group

\begin{tabular}{ll}
\hline \hline $\begin{array}{l}\text { Specialist Group Communication Ratings } \\
\text { Compared }\end{array}$ & t test p value \\
\hline Medical subspecialists vs. Surgical subspecialists & $\mathbf{0 . 0 0 0 0}$ \\
Medical subspecialists vs. Population specialists & $\mathbf{0 . 0 0 0 0}$ \\
Medical subspecialists vs. Other specialists & 0.0259 \\
Surgical specialists vs. Population specialists & $\mathbf{0 . 0 0 0 0}$ \\
Surgical specialists vs. Other specialists & $\mathbf{0 . 0 0 0 0}$ \\
Population specialists vs. Other specialists & $\mathbf{0 . 0 0 1 3}$ \\
\hline
\end{tabular}

Statistically significant values appear in bold. We adjusted for multiple comparisons, and the criteria for statistically significant differences was $0.05 / 6$, or 0.0083 . 
including negative data, as LVNs and clerks are not typically involved in communication with specialists. Of these 32 interviews, 20 ( $49 \%$ of the total sample) contained relevant content based on the participant's personal experience communicating with specialists.

Similar to the survey responses, interviews revealed that PCPs experience differences across specialties with respect to ease of communication. One PCP described this variability in primary-specialty communication around referrals: "There's no direct communication most of the time. It's by the consult [in the shared EMR]. It's what you write. To be perfectly honest, it is specialty-dependent. Some specialties are better than others."

PCPs and primary care staff involved in coordinating referrals reported substantial difficulty communicating with specialists about patient referrals. Challenges included difficulty communicating special requests for appointments and rejected referral requests.

Challenge: Difficulty Communicating Special Requests for Appointments. Difficulty communicating special requests via the EMR for appointments within needed time frames was reported by PCPs and primary care RNs. The automated nature of the EMR, with its lack of ability to triage or prioritize based on acuity, was described as contributing to this problem.

A PCP explained: "Sometimes [the patients] have to be seen sooner, and [the specialists] give me an appointment [through the EMR]. [I think] oh, that's way too long, I'll put a call into a fellow and see if I can do some kind of intervention before they see the patient if it's a specialty problem. And I'll tell them the problem: what can I do because he can't be seen until this time? Or I'll try to squeeze him in earlier, or sometimes I tell the patient just call each day: 'You have to call every day, and if there's a cancellation, they can get you in."

A primary care $\mathrm{RN}$ described a case where the clinical urgency of the patient's pain required an appointment with neurosurgery sooner than was assigned: "I have this one patient who [was referred to] neurosurgery clinic. He's having a lot of pain. And so the consult was booked [in the EMR]. It was March, and then the appointment, they just give it like June, so it's a long wait. So I kind of called them almost every day and bugged them, 'He's really in pain... 'So it's really hard... And sometimes you get upset because of this kind of system, but what can you do?"

Challenge: Rejected Referral Requests. PCP and primary care RN respondents described frustration with rejected referral requests, i.e., when a consult request placed in the EMR was rejected due to an administrative issue (e.g., clerical error) or incompleteness of information (e.g., not all consultassociated requirements were met).

One primary care $\mathrm{RN}$ described learning the requirements for consults as a "trial by fire": "That was probably the hardest to learn, because every time I make a consult, then it was denied. I said, 'Well, why was that denied?' So now I have to go back and find out why my consult for this patient was denied, because we didn't go through three steps first." A nurse commented that the burden of rejected referral requests combined with other referral delays created situations that could upset patients: "...You write the consult, and it could be several weeks before the consults are addressed and the patient's been seen, actually physically seen, or the consult gets kicked back to you. Now I have to call the patient and say, 'You know, we put that consult in. I'm really sorry, but we have to do a little homework first.' And I try to, you know, low-key it the best I can so the patients don 't get upset."

Rejected referral requests were also described as occurring when all of the requirements for a consult were not met, especially when PCPs and specialty providers disagreed about the importance of particular labs or tests, how recently they needed to be completed, if one test could stand in for another in its ability to measure a similar issue, or if a test needed to be repeated. In discussing this, one PCP described the relationship between primary and specialty as "really bad." He described the process of getting a consult with orthopedics, for example, as "next to impossible." Using urology as another example, he elaborated, “...There's so many barriers to consulting some specialists, and it's a hassle. A good example is a PSA [prostate-specific antigen] that's elevated. You know, you have to do all this and then order different PSA because [the specialists] want it. Well, it's like it's obvious it's too elevated. I mean how-what more PSA do you want? You know, if it's 40 or $50 \ldots$ there's no reason to do another one."

Despite these challenges, PCPs and primary care staff were able to identify ways in which the challenges could be mitigated, including phone and e-mail contact to supplement EMR communication, as well as electronic consultations, or "eConsults."

Response to Challenges: Phone and E-mail Contact to Supplement EMR Communication. PCPs indicated that other forms of referral communication were used to supplement the EMR in cases where EMR-based communication was not meeting a need. They reported using the phone or e-mail to contact specialty providers whom they knew in order to alert them to the needs of a particular patient and to request an appointment. This type of supplementary communication outside the EMR tended to be predicated on the PCP having an existing relationship with the specialist. One PCP explained: "I put in a [EMR] consult ...but there's this kind of lack of trust, I guess, between primary care and specialty clinic. So I actually made it a point to stay late and wrote a summary and e-mailed the department head, who I happen to know, and that way I made sure [the patient] got an appointment."

PCPs cited established relationships and trust between colleagues as key components to good communication with specialists around referrals. Close physical proximity, as in the case of the integration of mental health specialists into 
primary care clinics, was seen as facilitative of relationships and communication with specialists. One PCP described her interaction with a mental health specialist whose office was a few doors down the hall: "We have an excellent contact with the mental health provider. It's probably one of the best collaborations between mental health and primary care... The [core primary care] team is supposed to be the NP, the RN, the $L V N$, and the [clerk]. But I feel that he's part of the team, and it's just because it's just so fluid." Similarly, another PCP described the contrast between working in very close proximity with mental health specialists and communicating with specialty departments that were not co-located: "That's different than what we do with... our cardiologist or whatever. You just send something nebulously through the Internet [via the EMR] and hope that something happens on the other end. It does, and we get the consults back, but it's not like the personal connection that we just walk down the hall and talk to someone like it is with mental health."

Response to Challenges: eConsults. With regard to challenges with referral communication, respondents commented on how an innovation within the EMR, electronic consultations ("eConsults"), was able to facilitate communication between PCPs and specialists. eConsults allow PCPs to ask a discrete question related to a patient's management that the specialist may be able to answer electronically based on a review of the information contained in the EMR. Questions might include whether the patient needs a face-to-face visit with specialty care or the need and utility of relevant pre-visit laboratory tests.

One PCP praised the speed and responsiveness of eConsults: "Recently, there has been one positive development, which is the eConsult. So, eConsults tend to be very responsive right away because we don't need to see the patient. That is helpful. So, if you have a question-you don't think the patient needs to be seen, if you have a question, you do the eConsult, he responds right away. That's a recent thing...eConsults have been helpful."

Another PCP described the ease of use and efficiency of eConsults over the standard EMR-based referral: "One thing that specialists have started doing departmentally...is putting in like an eConsult, which is a phenomenal idea.... As before, you always just had to put in a standard consult, the patient would have to drive down, go in [to the office]. But now they're doing more eConsults, which is really nice... I just found out about it last week. So I think that it has the potential for really decreasing their backlog and facilitating - getting quicker answers to questions the primary care providers have."

\section{DISCUSSION}

Consistent with previous findings, our study showed that even in an integrated healthcare system with a shared EMR, primary-specialty communication was challenging, and more specifically, that PCPs faced issues with EMR-based referral communication. We found that such issues included difficulty requesting an appointment within a certain time frame as well as frequent rejected referral requests. To address these challenges, PCPs reported using phone and e-mail communication in cases where they had already established a relationship with the specialist, and took advantage of eConsults as an alternative to a traditional consult. Additionally, we found substantial variability by specialty with regard to PCPs' perception of ease of communication.

VHA PCPs and specialty providers can be seen as "dispersed collaborators,"26,27 which describes collaborators who are dispersed in geographic location (between two areas of a hospital or between a distant outpatient clinic and a VHA medical center), but who share a common goal of achieving optimal patient care. Dispersed collaborators strive to establish "mutual knowledge," 28 information that both parties need to have in common (e.g., an understanding of the patient's condition, reason for a referral, and/or a coordinated treatment plan for ongoing patient care), facilitated by electronic-based communication tools. Establishing mutual knowledge is more difficult for partners in dispersed versus face-to-face collaboration, however, due to the impersonal nature of technological communication, and dispersed collaborators are prone to miscommunication, which can be damaging to trust. ${ }^{26,27}$

It is not clear why we found substantial variability in PCP ratings of ease of communication among various specialties. We hypothesize that when PCPs and specialists have more opportunities for contact outside the EMR, they build face-to-face familiarity that facilitates communication. This hypothesis is substantiated by our finding that, on average, PCPs rated higher ease of communication with population specialists (including women's health and HIV/AIDS) and some other specialists (including mental health and rehabilitation) than the medical subspecialties; surgical subspecialists, on average, had the lowest ease-of-communication ratings. Women's health, one of two specialties rated the highest in ease of communication, is a primary care-oriented population specialty service, typically with strong links to the primary care clinic. Mental health, the other specialty with the highest ease-of-communication rating, is also closely tied with primary care due to the VHA's efforts to integrate mental health into primary care clinics. ${ }^{29}$ PCPs emphasized the importance of existing relationships between providers as facilitative of referral communication. Therefore, due to their close physical proximity, PCPs and mental health providers working in integrated primary care-mental health clinics may function more as face-to-face collaborators rather than dispersed collaborators, thereby facilitating communication.

Even in the context of a shared EMR, the PCP-specialist interface requires more than an exchange of information: it requires collaboration" 4,9 and agenuine dialogue" 23 based on personal interaction. ${ }^{17}$ Some evidence suggests that informal face-to-face contact between PCPs and specialist providers (passing each other in the hall, etc.) facilitates opportunities for communication $^{23}$ and may help to improve communication. ${ }^{30}$ 
For example, interactive interventions aimed at improving quality of communication between PCPs and specialty providers may have contributed to improved patient outcomes for diabetes and mental illness. ${ }^{31}$ This may explain our finding that eConsults, which create a virtual mechanism for these "hallway conversations," were viewed by PCPs as a positive development that is improving communication with specialists. Further, this need for interaction may explain why PCP-reported strategies to supplement EMR communication were modalities where they interacted more personally with specialists. Further data is needed with regard to the effects of eConsults and other innovations in improving $\mathrm{PCP}-$ specialist communication.

In order to address challenges associated with EMR-based referral communication, more flexible EMR platforms are needed. For example, EMRs might be improved to accommodate special cases, such as justification for appointment dates within particular time frames or exceptions to certain preconsult requirements such as repetitious labs, which could help to prevent rejected referral requests. An EMR more adept at managing a greater range of special cases may reduce the need for supplementary phone and e-mail communication. Based on the nature of strategies for supplementing EMRbased communication already in use, efforts to establish relationships between PCP and specialist providers may also facilitate improved referral communication. Because of the collaborative nature of referral communication and the highly case-specific nature of the mutual knowledge involved, some elements of direct physician-to-physician interface may always be necessary. Better relationships between dispersed PCPs and specialists may bridge the referral communication gaps in ways that EMRs cannot.

This study had notable limitations. While the survey attempted to obtain a census, non-responders (46\%) may have been different from responders (54\%) in some unmeasured way. We addressed survey non-response in quantitative analysis through the calculation and application of non-responder weights based on job title and site. Another limitation was the threat to external validity; the scope of the study was confined to one regional network, and may not be generalizable to other VHA networks or settings outside the VHA. However, an earlier national survey of VHA primary care directors found similar frequent $\mathrm{PC}-$-specialty interface issues ${ }^{32}$ (Danielle E. Rose, unpublished manuscript). Finally, the data used in this analysis are from the perspective of PCPs and others in primary care. Additional studies with specialists are needed in order to explore their perspectives on primary-specialty communication.

In conclusion, communication between primary and specialty care varies by specialty, and may be associated with the likelihood of an established connection already in place between specialty and primary care. When PCPs face challenges associated with communication via the EMR system, their strategies to supplement traditional EMR-based referral communication with other forms of communication provide clues as to the importance of relationships between PCPs and specialists. As the use of EMRs in the U.S. healthcare system becomes more common, there is an increased need for more studies of the EMR as a referral communication tool and its role in improving care coordination. Solutions to challenges in primary-specialty communication are needed in order to minimize the burden of frustration caused by difficulties in communication and to help optimize patient care between primary and specialty settings.

\section{ACKNOWLEDGMENTS:}

Contributors: We would like to thank Dr. Alexis K. Huynh for her statistical guidance, Mr. Andy Lanto for his programming assistance, Ms. Jill Darling for her work on the human subjects application and with the IRB interface, and Mr. John McElroy for his coordination. Drs. John Øvretveit, Ken Wells, and Jack Needleman provided helpful feedback on early drafts of the paper.

Funders: Funding for the VAIL-PCC PACT Demonstration Laboratory initiative was provided to Drs. Lisa V. Rubenstein, Elizabeth M. Yano, and Lisa Altman from the VHA Office of Patient Care Services (Project \#XVA 65-018). Dr. Yano's time was supported by a Senior Research Career Scientist Award from the VA HSR\&D Service (Project \#RCS 05195). The views expressed within are solely those of the authors, and do not necessarily represent the views of the Department of Veterans Affairs or the United States government.

IRB Approval: All procedures were approved by the institutional review boards of the Greater Los Angeles VA Healthcare System (2011070725 and 2011-030295) and the RAND Corporation (HSPC Project ID: 2010-0870).

Prior Presentations: A version of this paper was presented as an oral presentation at the Academy Health 2014 Annual Research Meeting in San Diego, CA, June 2014.

Conflict of Interest: The authors declare that they have no conflicts of interest.

Corresponding Author: Jessica L. Zuchowski, PhD, MPH; Center for the Study of Healthcare Innovation, Implementation and Policy, VA Greater Los Angeles Healthcare System, Building 25, 16111 Plummer Street, Sepulveda, CA 91343, USA (e-mail: jessica.zuchowski@va.gov).

\section{REFERENCES}

1. Cummins RO, Smith RW, Inui TS. Communication Failure in Primary Care. Failure of Consultants to Provide Follow-up Information. J Am Med Assoc. 1980;243(16): 1650-1652.

2. McPhee SJ, Lo B, Saika GY, et al. How Good is Communication Between Primary Care Physicians and Subspecialty Consultants? Arch Intern Med. 1984; 144(6): 1265-1268.

3. Byrd JC, Moskowitz MA. Outpatient Consultation: Interaction between the General Internist and the Specialist. J Gen Intern Med. 1987;2(2):93-98.

4. Forrest CB, Glade GB, Baker AE, et al. Coordination of Specialty Referrals and Physician Satisfaction with Referral Care. Arch Pediatr Adolesc Med. 2000;154(5):499-506.

5. Gandhi TK, Sittig DF, Franklin M, et al. Communication Breakdown in the Outpatient Referral Process. J Gen Intern Med. 2000;15:626-631.

6. Stille CJ, Primack WA, Savageau JA. Generalist-Subspecialist Communication for Children with Chronic Conditions: A Regional Physician Survey. Pediatrics. 2003;112(6):1314-1320.

7. Walsh J, Harrison JD, Young JM, et al. What are the Current Barriers to Effective Cancer Care Coordination? A Qualitative Study. Health Serv Res. 2010; 10:132.

8. Mehrotha A, Forrest CB, Lin CY. Dropping the Baton: Specialty Referrals in the United States. Milbank Q. 2011;89(1):39-68.

9. Stille CJ, Primack WA. Letter: Interspecialty Communication: Old Problem, New Hope? Arch Intern Med. 2011;171(14):1300. 
10. O'Malley AS, Reschovsky JD. Referral and Consultation Communication between Primary Care and Specialist Physicians: Finding Common Ground. Arch Intern Med. 2011;171(1):56-65.

11. Barnett ML, Song Z, Landon BE. Trends in Physician Referrals in the United States, 1999-2009. Arch Intern Med. 2012;172(2):163-170.

12. Taylor EF, Lake T, Nysenbaum J, Peterson G, Meyers D. Coordinating care in the medical neighborhood: critical components and available mechanisms. White Paper (Prepared by Mathematica Policy Research under Contract No. HHSA290200900019I TO2). AHRQ Publication No. 11-0064. Rockville: Agency for Healthcare Research and Quality; 2011.

13. Haggerty JL, Pineault R, Beaulieu M-D, et al. Practice Features Associated with Patient-Reported Accessibility, Continuity, and Coordination of Primary Health Care. Ann Fam Med. 2008;6(2):116-123.

14. Forrest CB. A Typology of Specialists' Clinical Roles. Arch Intern Med. 2009;169(11):1062-1068.

15. Fisher ES. Building a Medical Neighborhood for the Medical Home. N Engl J Med. 2008;359(12):1202-1205.

16. Katz MH. Editorial: How Can We Know So Little about Physician Referrals? Arch Intern Med. 2012;172(2): 100.

17. Pham HH. Good Neighbors: How Will the Patient-Centered Medical Home Relate to the Rest of the Health-Care Delivery System? J Gen Intern Med. 2010;25(6):630-4.

18. Hysong SJ, Esquivel A, Sittig DF, et al. Towards Successful Coordination of Electronic Health Record Based-Referrals: A Qualitative Analysis. Implement Sci. 2011;6:84.

19. Sinsky CA. The Patient-Centered Medical Home Neighbor: A Primary Care Physician's View. Ann Intern Med. 2011;154:61-62.

20. Stille CJ, McLaughlin TJ, Primack WA, et al. Determinants and Impact of Generalist-Specialist Communication about Pediatric Outpatient Referrals. Pediatrics. 2006;118(4):1341-1349.

21. Singh H, Esquivel A, Sittig DF, et al. Follow-up Actions on Electronic Referral Communication in a Multispecialty Outpatient Setting. J Gen Intern Med. 2010;26(1):64-9.
22. Sittig DF, Gandhi TK, Franklin M, et al. A Computer-Based Outpatient Clinical Referral System. Int J Med Inform. 1999;55:149-158.

23. Yee HF. The Patient-Centered Medical Home Neighbor: A Subspecialty Physician's View. Ann Intern Med. 2011;154:63-64.

24. Rosland AM, Nelson K, Sun H, Dolan ED, Maynard C, Bryson C, Stark R, Shear JM, Kerr E, Fihn SD, Schectman G. The patient-centered medical home in the Veterans Health Administration. Am J Manag Care. 2013;19(7):e263-72.

25. Rubenstein LV, Stockdale SE, Sapir N, et al. A patient-centered primary care practice approach using evidence-based quality improvement: rationale, methods, and early assessment of implementation. J Gen Intern Med. 2014;29(Suppl 2):S589-97.

26. Cramton CD. The Mutual Knowledge Problem and Its Consequences for Dispersed Collaboration. Organ Sci. 2001;12(3):346-371.

27. Cramton CD. Finding Common Ground in Dispersed Collaboration. Organ Dyn. 2002;30(4):356-367

28. Krauss RM, Fussell SR. Mutual knowledge and communicative effectiveness. In: Galegher J, Kraut RE, Egido C, eds. Intellectual Teamwork: Social and Technical Bases of Collaborative Work. Hillsdale: Erlbaum; 1990.

29. Chang ET, Rose DE, Yano EM, Wells KB, Metzger ME, Post EP, Lee ML, Rubenstein LV. Determinants of readiness for primary care-mental health integration (PC-MHI) in the VA Health Care System. J Gen Intern Med. 2013;28(3):353-62

30. Epstein RM. Communication between primary care physicians and consultants. Arch Fam Med. 1995;4(5):403-9.

31. Foy R, Hempel S, Rubenstein L, et al. Meta-analysis: Effect of Interactive Communication between Collaborating Primary Care and Specialists. Ann Int Med. 2010;152(4):

32. Yano EM, Fleming B, Canelo I, et al. National Survey Results for the Primary Care Director Module of the VHA Clinical Practice Organizational Survey. Technical Monograph \#08-CPOS02. Sepulveda: VA HSR\&D Center for the Study of Healthcare Provider Behavior; 2008. 